\title{
ON THE SPACE OF INTEGRAL FUNCTIONS. III
}

\section{GANAPATHY IYER}

1. Introduction. This paper consists of two parts. The properties of the space $\Gamma$ of integral functions were studied in $[1 ; 2]$. In part 1 of this paper, I prove three theorems on the closed linear subspace of $\Gamma$ spanned by specified classes of integral functions. In part 2, I study continuous linear transformations of $\Gamma$ into $\Gamma$.

2. Firstly we recall some of the main definitions and results proved ${ }^{1}$ in $[1 ; 2]$. The symbol $\Gamma$ denotes the class of all integral functions topologised by the metric $|\alpha-\beta|$ where $\alpha=\alpha(z)=\sum_{n=0}^{\infty} a_{n} z^{n}$, $\beta=\beta(z)=\sum_{n=0}^{\infty} b_{n} z^{n}$, and

$$
|\alpha-\beta|=\max \left[\left|a_{0}-b_{0}\right|,\left|a_{n}-b_{n}\right|^{1 / n}, n \geqq 1\right] .
$$

The space $\Gamma$ is a non-normable, complete, separable, linear metric space. The adjoint space $\Gamma^{*}$ of continuous linear functionals defined on $\Gamma$ is algebraically isomorphic to the class of all power series with positive radius of convergence so that each $f \in \Gamma^{*}$ is determined by a sequence

$$
\left(c_{n}\right) \text { with }\left\{\left|c_{n}\right|^{1 / n}\right\} \text { bounded }
$$

and $f(\alpha)=\sum_{n=0}^{\infty} c_{n} a_{n}, \alpha=\sum_{n=0}^{\infty} a_{n} z^{n}$. Sometimes it is convenient to write $f=f(z)=\sum_{0}^{\infty} c_{n} z^{n}$.

2.1. For each $R>0$, we denote by $\Gamma(R)$ the class of all integral functions topologised by the norm $|\alpha ; R|$ defined by

$$
|\alpha ; R|=\sum_{0}^{\infty}\left|a_{n}\right| R^{n}, \quad \alpha=\sum_{0}^{\infty} a_{n} z^{n} .
$$

Each element $f \in \Gamma^{*}(R)$ is determined by a sequence $\left(c_{n}\right)$ with $\left(\left|c_{n}\right| / R^{n}\right)$ bounded [2, p. 88]. If $E \subset \Gamma$, then the closure of $E$ in $\Gamma$ is the intersection of the closures of $E$ in $\Gamma(R)$ for $R>0$ and

$$
\Gamma^{*}=\sum_{R>0} \Gamma^{*}(R)
$$

[2, pp. 87-88]. We quote in the form of a lemma the main result used in proving the theorems mentioned above and those below.

Lemma. If $|\alpha| \geqq d>0$, then $|\alpha ; R| \geqq d$ for $R>A(1 / d)$ where $A(1 / d)$ is the greater of the two numbers 1 and $1 / d$.

Received by the editors March 7, 1952.

1 The numbers in brackets refer to the bibliography at the end. 
2.2. We have proved in [2, p. 89 , Theorems 3 and 4] that if $S$ is a linear subspace of $\Gamma$, then (1) every continuous linear functional defined on $S$ can be extended to the whole of $\Gamma$ so as to be continuous and linear and (2) if $\alpha \in \Gamma$ is at a positive distance from $S$, there is one $f \in \Gamma^{*}$ with $f(\alpha)=1$ and $f(\beta)=0$ for all $\beta \in S$. The result (2) stated above leads immediately to the following theorem which will be used to derive the results in part 1 . In this theorem for a set $E \subset \Gamma$ the symbol $L\{E\}$ stands for the closed linear subspace of $\Gamma$ generated by the elements of $E$.

Theorem 1. Let $E \subset \Gamma$. An element $\alpha \in \Gamma$ will belong to $L\{E\}$ if and only if $f(\alpha)=0$ for every $f \in \Gamma^{*}$ such that $f(\beta)=0$ for all $\beta \in E$.

\section{PART 1}

3. ThEOREM 2. Let $\alpha=\alpha(z)=\sum_{n=0}^{\infty} a_{n} z^{n}$ be an element of $\Gamma$ such that no coefficient $a_{n}$ is zero. Let $z_{n}, n=1,2, \cdots$, be a sequence of distinct complex numbers. Let $\alpha_{n}=\alpha\left(z z_{n}\right)$. Let one of the following conditions be satisfied:

(i) The sequence $\left(z_{n}\right)$ has a finite limit point;

(ii) $\alpha$ is of order $p$ and finite type and $\lim \sup _{n \rightarrow \infty} n /\left|z_{n}\right|^{p}=\infty$. Then $L\left\{\alpha_{n}, n \geqq 1\right\}=\Gamma$.

Proof. Let $f=\sum_{0}^{\infty} c_{n} z^{n} \in \Gamma^{*}$ and let $f\left(\alpha_{n}\right)=0, n=1,2, \cdots$ Then we get

$$
\sum_{p=0}^{\infty} c_{p} a_{p} z_{n}^{p}=0, \quad n=1,2, \cdots .
$$

Now $g(z)=\sum_{p=0}^{\infty} c_{p} a_{p} z^{p}$ is always an integral function and if (ii) is true, it is an integral function of order $p$ and finite type. Since $g\left(z_{n}\right)=0$, we see from classical theorems on integral functions [3] that $g(z) \equiv 0$ so that $c_{p} a_{p}=0$ and since no $a_{p}=0$, we get $c_{p}=0$ for $p=0,1,2, \cdots$. Hence $f$ is the identically zero functional. So by Theorem 1 every $\alpha \in \Gamma$ is in $L\left\{\alpha_{n}, n \geqq 1\right\}$. This proves the theorem.

3.1. REMARK. If some of the coefficients of $\alpha=\sum_{0}^{\infty} a_{n} z^{n}$ be zero, the same argument shows that under the hypothesis (i) or (ii) of Theorem $2 L\left\{\alpha_{n}\right\}$ will be the same as the closed linear subspace of $\Gamma$ spanned by those powers of $z$ in $\alpha$ whose coefficients do not vanish.

3.2. Illustrations. Every integral function could be obtained as the uniform limit (in any finite circle $[1$, p. 18, Theorem 3]) of finite linear combinations selected from each of the following sequences:

(1) $\alpha(z / n), n=1,2, \cdots$, where no coefficient is zero in $\alpha$ $=\sum_{0}^{\infty} a_{n} z^{n}$.

(2) $e^{z n^{1 / 2}}, n=1,2, \cdots$ 
(3) $\cos 2 \pi z n^{1 / 2}+\sin 2 \pi z n^{1 / 2}, n=1,2, \cdots$.

4. If $\alpha=\sum_{0}^{\infty} a_{n} z^{n}, \beta=\sum_{0}^{\infty} b_{n} z^{n}$, we define $\alpha \circ \beta$ by

$$
\alpha \circ \beta=\sum_{0}^{\infty} a_{n} b_{n} z^{n}
$$

We denote by $(\alpha)_{n}$ the function $\alpha \circ \alpha \circ \alpha \circ \cdots \circ \alpha, n$ times.

Theorem 3. Let $\alpha=\sum_{0}^{\infty} a_{n} z^{n} \in \Gamma$ be such that $\operatorname{Re}\left(a_{n}\right)$ is a strictly decreasing sequence. Then $L\left\{(\alpha)_{n}, n \geqq 1\right\}$ is equal to $\Gamma$.

Proof. Let $f=\sum_{p=0}^{\infty} c_{p} z^{p} \in \Gamma^{*}$ be such that $f\left[(\alpha)_{n}\right]=0, n$ $=1,2, \ldots$. This gives the relations

$$
\sum_{p=0}^{\infty} c_{p} a_{p}^{n}=0, \quad n=1,2,3, \cdots .
$$

Let

$$
g(z)=\sum_{p=0}^{\infty} c_{p} a_{p} e^{a_{p} z}
$$

Since $\sum\left|c_{p} a_{p}\right|$ converges by (2) and $a_{p} \rightarrow 0$ as $p \rightarrow \infty$, we see that $g(z)$ is an integral function. By $(6), g^{(n)}(0)=0, n=0,1,2, \cdots$. Hence $g(z) \equiv 0$. If $\lambda>0$, we have

$$
0=\int_{0}^{\lambda} g(z) e^{-a_{0} z} d z=\lambda c_{0} a_{0}+\sum_{p=1}^{\infty} c_{p} a_{p} \int_{0}^{\lambda} e^{-\left(a_{0}-a_{p}\right) z} d z
$$

In virtue of the hypothesis on $\operatorname{Re}\left(a_{n}\right)$ the expression

$$
\sum_{p=1}^{\infty} c_{p} a_{p} \int_{0}^{\lambda} e^{-\left(a_{0}-a_{p}\right) z} d z
$$

is bounded as $\lambda \rightarrow \infty$. So (8) gives $c_{0} a_{0}=0$ and so $c_{0}=0$ (since no $a_{n}$ is zero by hypothesis). Repeating the argument and using induction we see that $c_{n}=0$ for $n=0,1,2, \ldots$. Therefore $L\left\{(\alpha)_{n}, n \geqq 1\right\}=\Gamma$ as in the previous theorem.

4.1. REMARK 1 . It is not necessary to suppose that $\operatorname{Re}\left(a_{n}\right)$ strictly decreases. It is enough to suppose that $\operatorname{Re}\left(a_{0}\right) \geqq \operatorname{Re}\left(a_{1}\right) \geqq \operatorname{Re}\left(a_{2}\right)$ $\geqq \cdots$, the $a_{n}$ 's are all distinct from one another, and that no $a_{n}$ is zero. This will follow from the above proof if we observe that for $t$ real and not zero

$$
\left|\int_{0}^{\lambda} e^{i t x} d x\right| \leqq \frac{2}{|t|}, \quad \lambda>0 .
$$


4.2. REMARK 2. Instead of supposing that the real parts of $a_{n}$ satisfy the conditions of Theorem 3 or those of $\$ 4.1$, we can suppose that for some real $\theta$, the sequence $a_{n} e^{i \theta}$ has the same property.

4.3. Illustrations. Theorem 3 applies to the following functions:

$$
\begin{aligned}
e^{z}-\frac{1}{3} z & =1+\frac{2}{3} z+\frac{z^{2}}{2 !}+\frac{z^{3}}{3 !}+\cdots, \\
\cosh z^{1 / 2} & =1+\frac{z}{2 !}+\frac{z^{2}}{4 !}+\cdots, \\
\alpha(z) & =2+\frac{z}{1}+\frac{z^{2}}{2^{2}}+\cdots+\frac{z^{n}}{n^{n}}+\cdots,
\end{aligned}
$$

but does not apply to $e^{z}$, the first two coefficients being equal. It can be directly verified that there exist $f \in \Gamma^{*}$ not identically zero such that $f\left[\left(e^{z}\right)_{n}\right]=0, n=1,2,3, \cdots$.

5. Theorem 4. Let $Z$ denote a set of complex numbers. Let for $\alpha \in \Gamma$ the symbol $(\alpha, Z)$ denote the set of functions $\alpha(z+\lambda), \lambda \in Z$. Let $\alpha^{(p)}$ denote as usual the pth derivative of $\alpha$. The subspaces $L\{(\alpha, Z)\}$ and $L\left\{\alpha^{(p)}, p \geqq 0\right\}$ are the same provided one of the following conditions be satisfied:

(i) The set $Z$ has a finite limit point;

(ii) $\alpha$ is of order $\rho$ and finite type and $Z$ contains a sequence $z_{n}$ of distinct points such that $\lim \sup _{n \rightarrow \infty} n /\left|z_{n}\right|^{\rho}=\infty$.

Proof. Let $\alpha=\sum_{0}^{\infty} a_{n} z^{n}$. Then

$$
\alpha^{(p)}=\sum_{n=0}^{\infty}(n+1)(n+2) \cdots(n+p) a_{p+n} z^{n} .
$$

So if $f=\sum_{0}^{\infty} c_{n} z^{n} \in \Gamma^{*}$ be such that $f\left[\alpha^{(p)}\right]=0, p=0,1,2, \cdots$, we get

(9) $A_{p}=\sum_{n=0}^{\infty}(n+1)(n+2) \cdots(n+p) c_{n} a_{p+n}=0, p=0,1,2, \cdots$. If $f[\alpha(z+\lambda)]=0$ for $\lambda \in Z$, then

$$
\sum_{p=0}^{\infty} c_{p} \frac{\alpha^{p}(\lambda)}{p !}=0 \quad \text { for } \lambda \in Z .
$$

Using the expression for $\alpha^{(p)}(\lambda)$ in (10), using the classical inequalities for the coefficients in a power series, and noting that the double series involved in the necessary rearrangement is absolutely convergent, we see that (10) is equivalent to 


$$
\sum_{p=0}^{\infty} A_{p} \frac{\lambda^{p}}{p !}=0,
$$

The series in (11) converges for all $\lambda$ and so under (i) of the theorem (9) and (10) are equivalent so that the theorem follows from Theorem 1 in this case. To prove the same under (ii) of the theorem we have to show that $\sum A_{p} Z^{p} / p$ ! is an integral function of order $\rho$ and finite type. To do this it is enough to prove that $\left.p^{1 / p}\left|A_{p} / p !\right|\right|^{1 / p}$ is bounded $\left[3\right.$, p. 41]. Now by (2) there is a $K_{1}$ such that $\left|c_{n}\right| \leqq K_{1}^{n+1}$, $n=0,1,2, \cdots$. Since $\alpha$ is of order $\rho$ and finite type, there is $k_{2}$ such that $n^{1 / p}\left|a_{n}\right|^{1 / n} \leqq K_{2}, n=1,2, \cdots$. If $p \geqq 1$, we have

$$
\begin{aligned}
\left|A_{p}\right| & \leqq \sum_{n=0}^{\infty}(n+1) \cdots(n+p)(n+p)^{-(n+p) / p} K_{1}^{n+1} K_{2}^{n+p} \\
& \leqq K_{1} K_{2}^{p} p^{-p / p} \sum_{n=0}^{\infty}\left(K_{1} K_{2}\right)^{n}(n+p)^{p-n / p} .
\end{aligned}
$$

Let $t$ be the integral part of $\rho p$. If $p$ is large and $0 \leqq n \leqq t$, it is easily seen that $(n+p)^{p-n / p} \leqq p^{p}$. Hence

$$
\begin{aligned}
\left|A_{p}\right| \leqq K_{1} K_{2}^{p} p^{p-p / p} \sum_{n=0}^{t} & \left(K_{1} K_{2}\right)^{n} \\
& \quad+K_{1} K_{2}^{p}\left(K_{1} K_{2}\right)^{t} p^{-p / p} \sum_{n=1}^{\infty}\left(K_{1} K_{2}\right)^{n} n^{-(n+t-p \rho) / p} .
\end{aligned}
$$

Since $|t-p \rho| \leqq 1$, we see from the above that $p^{1 / p}\left|A_{p} / p !\right|^{1 / p}$ is bounded as $p \rightarrow \infty$ noting that $(a+b)^{1 / p} \leqq a^{1 / p}+b^{1 / p}, a, b>0$ and $\left[p^{p}(p !)^{-1}\right]^{1 / p}=O(1)$ as $p \rightarrow \infty$. This completes the proof of the theorem.

5.1. Illustrations. Consider $\alpha=e^{z^{2}}$. Here $\alpha^{(n)}=e^{z^{2}} Q_{n}(z)$ where $Q_{n}(z)$ is a polynomial of precise degree $n$. Since $z^{n}, n=0,1,2, \cdots$, can be expressed as a finite linear combination of $Q_{0}(z), Q_{1}(z), \cdots$, and every integral function could be put in the form $e^{z^{2}} \beta$ where $\beta$ is an integral function, it follows that $L\left\{\alpha^{(n)}, n \geqq 0\right\}=\Gamma$. Hence the set $L\left\{e^{\left(z+n^{-1}\right)^{2}}, n \geqq 1\right\}$ or the set $L\left\{e^{\left(z+n^{1 / 3}\right)^{2}}, n \geqq 1\right\}$ is the whole space $\Gamma$. Note that if $\alpha=e^{z}$, then $L\left\{\alpha^{(n)}, n \geqq 0\right\}$ is merely the onedimensional subspace of constant multiples of $e^{z}$.

\section{PART 2}

6. We now consider continuous linear transformations whose domain is the whole of $\Gamma$ and whose range is in $\Gamma$. When the range is also the whole of $\Gamma$, we use the usual term "onto." The main result 
in this connection is that every such continuous linear transformation can be specified in terms of a family of continuous linear transformations of normed spaces into normed spaces. We denote by $T\left(R_{1} \rightarrow R_{2}\right)$ a continuous linear transformation whose domain is $\Gamma\left(R_{1}\right)$ and range is in $\Gamma\left(R_{2}\right)$. We denote the family of such transformations by $F\left(R_{1} \rightarrow R_{2}\right)$. Consistent with this notation we denote by $T(\infty \rightarrow \infty)$ a continuous linear transformation of $\Gamma$ into $\Gamma$ and the family of such transformations by $F(\infty \rightarrow \infty)$.

7. THEOREM 5. The following relation is valid:

$$
F(\infty \rightarrow \infty)=\prod_{R_{2}>0}\left\{\sum_{R_{1}>0} F\left(R_{1} \rightarrow R_{2}\right)\right\} .
$$

In other words, each $T(\infty \rightarrow \infty)$ is a $T\left(R_{1} \rightarrow R_{2}\right)$ for each $R_{2}>0$ and a corresponding suitably chosen $R_{1}>0$.

Proof. The topology $\Gamma(R)$ becomes weaker as $R$ increases (in the sense of $[4$, p. 62$])$ and $\Gamma$ is the topology just weaker than all the $\Gamma(R), R>0[2, \mathrm{p} .87])$. By known properties of stronger and weaker topologies $[5$, p. 71$]$ it follows that a $T(\infty \rightarrow \infty)$ is a $T\left(\infty \rightarrow R_{2}\right)$ for each $R_{2}>0$. Hence

$$
F(\infty \rightarrow \infty) \subset \prod_{R_{2}>0} F\left(\infty \rightarrow R_{2}\right) .
$$

Now suppose that a linear transformation $T$ of $\Gamma$ into $\Gamma$ is not continuous. Then there exists a sequence $\left(\alpha_{p}\right)$ of elements of $\Gamma$ such that $\left|\alpha_{p}\right| \rightarrow 0$ as $p \rightarrow \infty$ but $\left|T\left(\alpha_{p}\right)\right| \geqq d>0, p=1,2, \cdots$. By the lemma of $\$ 2.1$, we see that $\left|T\left(\alpha_{p}\right) ; R\right| \geqq d$ for $R>A(1 / d)$, that is, $T$ is not a $T\left(\infty \rightarrow R_{2}\right)$ for $R_{2}>A(1 / d)$. This along with (12) proves that

$$
F(\infty \rightarrow \infty)=\prod_{R_{2}>0} F\left(\infty \rightarrow R_{2}\right) .
$$

Now let $R_{2}>0$ be fixed. Any $T\left(R_{1} \rightarrow R_{2}\right), R_{1}>0$, is a $T\left(\infty \rightarrow R_{2}\right)$. Hence

$$
\sum_{R_{1}>0} F\left(R_{1} \rightarrow R_{2}\right) \subset F\left(\infty \rightarrow R_{2}\right) .
$$

Suppose that a linear transformation $T$ of $\Gamma\left(R_{1}\right)$ into $\Gamma\left(R_{2}\right)$ is not continuous for any $R_{1}>0$. Then by known properties of normed spaces $[6$, p. 54] we can, for each positive integer $p$, find an element $\alpha_{p}$ of $\Gamma(p)$ such that $\left|\alpha_{p} ; p\right| \leqq 1 / p$ while $\left|T\left(\alpha_{p}\right) ; R_{2}\right| \geqq 1$. From the definitions of $|\alpha ; R|$ and $|\alpha|$, it is easily verified that $\left|\alpha_{p}\right| \leqq 1 / p$ and so $\left|\alpha_{p}\right| \rightarrow 0$ as $p \rightarrow \infty$, while $\left|T\left(\alpha_{p}\right) ; R_{2}\right| \geqq 1$. This proves that such a $T$ is not a $T\left(\infty \rightarrow R_{2}\right)$. This along with (14) proves that 


$$
\sum_{R_{1}>0} F\left(R_{1} \rightarrow R_{2}\right) \subset F\left(\infty \rightarrow R_{2}\right) .
$$

The theorem follows from (13) and (15).

8. We write $\delta_{n} \equiv z^{n}, n=0,1,2, \cdots$. Theorem 4 leads to the following result.

TheOREM 6. A necessary and sufficient condition that there exists a $T=T(\infty \rightarrow \infty)$ with $T\left(\delta_{n}\right)=\alpha_{n}, n=0,1,2, \cdots$, is that, for each $R>0$, the sequence $\left|\alpha_{n} ; R\right|^{1 / n}$ is bounded.

Proof. $T \in F(\infty \rightarrow \infty)$ with $T\left(\delta_{n}\right)=\alpha_{n}, n=0,1,2, \cdots$. Then by Theorem 4, for each $R>0$, there is an $R_{1}>0$ such that $T \in F\left(R_{1} \rightarrow R\right)$. Hence by known properties of transformations between normed spaces $[6$, p. 54, Theorem 1], there is a $K=K(R)$ such that

$$
\left|T\left(\delta_{n}\right) ; R\right|=\left|\alpha_{n} ; R\right| \leqq K\left|\delta_{n} ; R_{1}\right|=K R_{1}^{n} .
$$

This proves that the condition is necessary. Conversely let the condition of the theorem be satisfied by the sequence $\left(\alpha_{n}\right)$ of elements of $\Gamma$. If $\alpha=\sum_{n=0}^{\infty} a_{n} \delta_{n}$, then $\left|a_{n}\right|^{1 / n} \rightarrow 0$ as $n \rightarrow \infty$. Hence the series $\sum a_{n} \alpha_{n}$ converges in $\Gamma$ to an element of $\Gamma[1$, p. 18, Theorem 3]. Now define $T(\alpha)=\sum_{0}^{\infty} a_{n} \alpha_{n}$ for $\alpha \in \Gamma$. Then $T\left(\delta_{n}\right)=\alpha_{n}, n=0,1,2, \cdots$, and for each $R>0$ we have $|T(\alpha) ; R| \leqq K \cdot\left|\alpha ; R_{1}\right|,{ }^{2}$ that is, $T \in F\left(R_{1} \rightarrow R\right)$. So by Theorem 4, $T \in F(\infty \rightarrow \infty)$. This completes the proof of the theorem.

8.1. Remark. If $\alpha_{n}=T\left(\delta_{n}\right), n=0,1,2, \cdots$, satisfies the condition of Theorem 6 , there is one and only one transformation of $F(\infty \rightarrow \infty)$ satisfying $T\left(\delta_{n}\right)=\alpha_{n}$. This does not preclude the existence of discontinuous linear transformations $T^{\prime}$ with $T^{\prime}\left(\delta_{n}\right)=\alpha_{n}$. By using any Hamel basis containing $\left(\delta_{n}\right)$ we can always construct such discontinuous transformations.

9. Automorphisms of $\Gamma$ and bases. We have defined a base of $\Gamma$ as a sequence $\alpha_{n}, n=0,1,2, \cdots$, of elements of $\Gamma$ such that every $\alpha \in \Gamma$ can be uniquely represented as a convergent series

$$
\alpha=\sum_{0}^{\infty} t_{n}(\alpha) \alpha_{n}
$$

[2, p. 92]. If, in (16), $\left|t_{n}(\alpha)\right|^{1 / n \rightarrow 0}$ as $n \rightarrow \infty$ for every $\alpha \in \Gamma$, then we shall call the base $\left(\alpha_{n}\right)$ a proper base (for instance the bases of [2,

${ }^{2}$ Here $K$ and $R_{1}$ are the numbers for which $\left|\alpha_{n} ; R\right| \leqq K R_{1}^{n}, n=0,1,2, \cdots$. Such numbers exist by the hypothesis on $\left(\alpha_{n}\right)$. 
p. 93, Theorem 8] are all proper). The following theorem gives the relations between automorphisms of $\Gamma$ and bases.

THEOREM 7. If $T$ is an automorphism (that is, a bi-uniform, bicontinuous, linear transformation of $\Gamma$ onto $\Gamma)$, then every base is transformed into a base; in particular $T\left(\delta_{n}\right), n=0,1,2, \cdots$, will be a base. Let $T$ be a transformation of $F(\infty \rightarrow \infty)$ such that $T\left(\delta_{n}\right)$ form a base. Then $T$ will be an automorphism if one of the following conditions be satisfied:

(i) $T$ is a transformation of $\Gamma$ onto $\Gamma$.

(ii) $T$ is a closed transformation, that is, takes closed sets in $\Gamma$ into closed sets in $\Gamma$.

(iii) The base $T\left(\delta_{n}\right)$ is a proper base.

Proof. The first part of the theorem is an easy consequence of the definition of automorphisms and bases. To prove the second part, let $T(\Gamma)$ denote the range of $T$. If $T\left(\delta_{n}\right)$ is a base, we show that $T$ transforms $\Gamma$ onto $T(\Gamma)$ in a one-to-one manner. If $\alpha=\sum a_{n} \delta_{n}$, $\beta=\sum b_{n} \delta_{n}$, then, since $T$ is continuous, we get $T(\alpha)=\sum a_{n} T\left(\delta_{n}\right)$ and $T(\beta)=\sum b_{n} T\left(\delta_{n}\right)$. If $T(\alpha)=T(\beta)$, we see from the definition of a base that $a_{n}=b_{n}, n=0,1,2, \cdots$, and therefore $\alpha=\beta$. Now if we know $T(\Gamma)=\Gamma$, then by a known theorem [6, p. 41, Theorem 5$]$ the inverse transformation (which exists as just now shown) is also continuous and obviously linear. So $T$ will be an automorphism. So we have to show that under the conditions of the theorem $T(\Gamma)=\Gamma$. In case (i) this is true by hypothesis. In case (ii), since $T\left(\delta_{n}\right)$ is a base, we have for every $\alpha \in \Gamma$

$$
\alpha=\sum t_{n}(\alpha) \alpha_{n}=\lim _{n \rightarrow \infty} T\left[\sum_{p=0}^{n} t_{p}(\alpha) \delta_{p}\right],
$$

so that $T(\Gamma)$ is dense in $\Gamma$. Since $T$ is closed, $T(\Gamma)$ must be closed in $\Gamma$ and so $T(\Gamma)=\Gamma$. In case (iii), we have for $\alpha \in \Gamma$

$$
\alpha=\lim _{n \rightarrow \infty} T\left[\sum_{p=0}^{n} t_{p}(\alpha) \delta_{p}\right] .
$$

Since the base is proper, $\sum_{p=0}^{n} t_{p}(\alpha) \delta_{p}$ converges to an element $\beta \in \Gamma$. Hence $T(\Gamma)=\Gamma$ in this case also. This completes the proof.

9.1. REMARK 1. The above theorem shows that the class of closed transformations $T$ of $F(\infty \rightarrow \infty)$ for which $T\left(\delta_{n}\right)$ form a base coincide with the class of automorphisms.

9.2. REMARK 2. I have not been able to prove or disprove the existence of improper bases. Nor is it known that every base $\left(\alpha_{n}\right)$ 
satisfies the condition that $\left|\alpha_{n} ; R\right|^{1 / n}$ is bounded for each $R>0$. It is likely that every base is proper and satisfies the above condition in addition. If this be so, the previous theorem shows that there will be a one-to-one correspondence between bases in $\Gamma$ and automorphisms of $\Gamma$.

10. Multiplicative transformations. A transformation $T \in F(\infty \rightarrow \infty)$ is said to be multiplicative if $T(\alpha \beta)=T(\alpha) T(\beta)$ for $\alpha, \beta \in \Gamma$. The following theorem gives a complete characterisation of such transformations.

TheOREM 8. Let $T \in F(\infty \rightarrow \infty)$ and $T\left(\delta_{1}\right)=\alpha$. Then if $T$ is not identically zero, it is multiplicative if and only if $T\left(\delta_{n}\right)=\alpha^{n}, n$ $=0,1,2, \cdots$ Moreover $T(\beta)=\beta[\alpha(z)], \beta=\beta(z) \in \Gamma$.

Proof. Let $T$ be not identically zero. Then the equation $T(\alpha)$ $=T(\alpha) T\left(\delta_{0}\right)$ shows that $T\left(\delta_{0}\right)=1$. From the equation $\delta_{m+n}=\delta_{m} \delta_{n}$ we see that $T\left(\delta_{n}\right)=\left[T\left(\delta_{1}\right)\right]^{n}, n=0,1,2, \cdots$. So if the transformation is multiplicative, the condition of the theorem is satisfied. Conversely let a $T \in F(\infty \rightarrow \infty)$ satisfy the condition $T\left(\delta_{n}\right)=\left[T\left(\delta_{1}\right)\right]^{n}$, $n=0,1,2, \cdots$. Then if $\alpha=\sum a_{n} \delta_{n}$, then $T(\alpha)=\sum a_{n}\left[T\left(\delta_{1}\right)\right]^{n}$ and so $T$ is multiplicative.

10.1. REMARK. If the multiplicative transformation is an automorphism, then $\left[T\left(\delta_{1}\right)\right]^{n}$ will form a base. But as indicated elsewhere $\left[2\right.$, p. 95] the only base of the form $\alpha^{n}, n=0,1,2, \cdots$, is when $\alpha=a z+b, a \neq 0$. Since this base is proper, the converse is also true by Theorem 7 . Hence the class of multiplicative automorphisms are of the form $T[\alpha(z)]=\alpha(a z+b), a \neq 0$.

11. Conclusion. From Theorem 6 we see that for a $T \in F(\infty \rightarrow \infty)$ the quantity

$$
\sigma(R ; T)=\max \left[\left|T\left(\delta_{0}\right) ; R\right|,\left|T\left(\delta_{n}\right) ; R\right|^{1 / n}, n \geqq 1\right]
$$

is bounded for each $R>0$. If we write

$$
\left|T_{1}-T_{2}\right|=\sum_{k=1}^{\infty} \frac{1}{2^{k}} \frac{\sigma\left(k ; T_{1}-T_{2}\right)}{1+\sigma\left(k ; T_{1}-T_{2}\right)}
$$

the expression defines a complete metric topology on $F(\infty \rightarrow \infty)$. In this topology $T_{1} \pm T_{2}$ is continuous and $T_{n} \rightarrow T$ as $n \rightarrow \infty$ implies that $T_{n}(\alpha) \rightarrow T(\alpha)$ for all $\alpha \in \Gamma$. But neither $c T$ nor $T_{1} T_{2}$ is continuous in this topology. The expression $\sigma(R ; T)$ itself can sometimes be used to give more information about the nature of $T$. For instance, $\sigma(R ; T)=O(1)$ as $R \rightarrow \infty$ if and only if $T\left(\delta_{n}\right)=c_{n} \delta_{0}$, where $c_{n}$ is a 
constant with $\left|c_{n}\right|^{1 / n}$ bounded, so that the class of such transformations is isomorphic (algebraically) to $\Gamma^{*}$. If $\sigma(R ; T)=O\left(R^{\rho}\right)$, then $T\left(\delta_{n}\right)$ is a polynomial of degree not exceeding $n \rho(\rho$ when $n=0)$. If $\log \sigma(R ; T)=O\left(R^{\rho}\right)$, then each $T\left(\delta_{n}\right)$ is an integral function of order $\rho$ and finite type at most. Finally it may be noted that if, for a linear $T$, the functions $T\left(\delta_{n}\right)=\alpha_{n}$ do not satisfy the condition of Theorem 6 , then $T \notin F(\infty \rightarrow \infty)$. For instance, if $T\left(\delta_{n}\right)=e^{n^{2} z}$ or $=\cos \left(n^{3 / 2} z\right)$ or $=z^{n^{2}}$, such a $T$ cannot be continuous.

\section{BibLIOGRAPHY}

1. V. Ganapathy Iyer, On the space of integral functions. I, J. Indian. Math. Soc. (2) vol. 12 (1948) pp. 13-30.

2. - On the space of integral functions. II, Quart. J. Math. Oxford Ser. (2) vol. 1 (1950) pp. 86-96.

3. G. Valiron, Lectures on the general theory of integral functions.

4. P. Alexandroff and H. Hopf, Topologie. I, Berlin, 1935.

5. R. Vaidyanathaswamy, Treatise on set topology.

6. S. Banach, Theorie des operations lineaires, Warsaw, 1932.

Annamalai University 